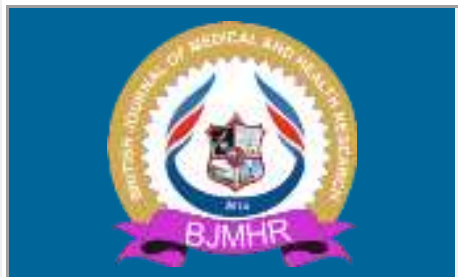

\title{
BJMHR
}

British Journal of Medical and Health Research Journal home page: www.bjmhr.com

\section{Incidental Thyroid Carcinoma in Thyroidectomy Specimens and Associated Risk Factors}

\author{
Bimalka Seneviratne \\ Department of Pathology / Cancer Research Centre, Faculty of Medical Sciences, \\ University of Sri Jayewardenepura, Sri Lanka
}

\section{ABSTRACT}

Thyroid cancer has shown an upward trend in most parts of the world during the last few decades. In Sri Lanka thyroid malignancy is the second most common cancer in females. Incidental thyroid cancer is an unexpected malignancy seen following thyroidectomy surgery. Aim of the study was to determine the frequency of incidental thyroid carcinoma in thyroidectomy specimens submitted for presumed benign pathological disease entities such as multinodular goiter, toxic nodular goiter, chronic thyroiditis and Grave's disease and identify the associated risk factors for the development of malignancy. A retrospective study which included 92 cases of thyroidectomy specimens from 3 specialized surgical centers. The cases with a prior fine needle aspiration cytology (FNAC) diagnosis of malignancy or suspicious of malignancy were excluded from the current study. The frequency of incidental thyroid carcinoma was $9.78 \%$ (09 out of 92 cases). All the cases were confirmed histologically as papillary carcinoma $(\mathrm{p}<0.05)$ and the majority were $<10 \mathrm{~mm}$ in diameter (microcarcinoma). Incidental thyroid carcinoma showed a statistically significant association with chronic thyroiditis $(p<0.05)$. With limited facilities for radiological evaluation and identification of suspicious nodules in the growing numbers of patients with multinodular goiters, the risk of missing incidental thyroid carcinoma especially microcarcinomas, will continue to lurch in the same manner.

Keywords: Incidental thyroid carcinoma, thyroidectomy, multinodular goiter 


\section{INTRODUCTION}

Diseases of thyroid result in enlargement of the gland (goiter) and may be associated with altered functions leading to hypothyroidism or a hyper functioning gland $(1,2)$. The prevalence of thyroid diseases in Sri Lanka has greatly increased over the last few decades due to multiple factors such as environmental hazards, dietary patterns, genetic alterations and radiation effects (3). Widened access for health care facilities and awareness programs on cancer have significantly improved the health seeking behaviour of individuals, thus contributing to the upward trend in thyroid case detection. According to cancer incidence data compiled by the national cancer control program of Sri Lanka (4), thyroid cancer is the second most common malignancy in females.

Most common cause of goiter is dietary iodine deficiency (5). As a consequence of iodine deficiency there is compensatory hyperplasia of thyroid gland (6). Increase in the functional mass of thyroid tissue helps to overcome the thyroid hormone deficiency and ensure an euthyroid state in the majority of individuals. This process leads to goitrous enlargement of thyroid which could occur in a diffuse manner (simple goiter) or in an uneven pattern, eventually resulting in a multinodular goiter (MNG), over a period of time (7). Both patterns of goiters could arise in an endemic setting or as a sporadic disorder (8).

Fine needle aspiration cytology (FNAC) technique is a rapid, cost effective and reliable first line investigation for thyroid disorders, widely accepted in developing countries including Sri Lanka. Majority of the patients with thyroid diseases would be subjected to this investigation prior to surgical intervention (9). FNAC procedure would provide a safe diagnosis in most of the instances with the expertise of an experienced pathologist (10). However, there are limitations when interpreting FNAC results and in a minority of cases could give rise to false positives and false negatives. Radiological findings also reveal valuable information in relation to goiters and both the above methods would complement each other in arriving at an accurate diagnosis (11). Most definitive information about the nature of the goiter is obtained by morphological assessment of the resected specimen. Multinodular goiters are less likely to be malignant when compared to solitary thyroid nodules.

Incidental thyroid carcinoma is an unexpected finding identified following histopathological evaluation of thyroidectomy specimens sent for presumed benign diseases such as multinodular goiter (MNG), chronic thyroiditis, Grave's disease or toxic nodular goiter. In the majority of patients of the study (> 80\%) the FNAC diagnosis has been that of a benign lesion and falling within Bethesda category II (12), of the thyroid cytology reporting process. In a minority of patients, the FNAC findings were inconclusive. Radiological findings have been suggestive of benign disease entities. 


\section{Objectives:}

To determine the frequency of incidental thyroid carcinoma in thyroidectomy specimens submitted for presumed benign pathological disease entities such as multinodular goiter, toxic nodular goiter, chronic thyroiditis and Grave's disease and identify the associated risk factors for the development of malignancy.

\section{MATERIALS AND METHOD}

A retrospective analysis of the medical records of patients who underwent thyroidectomy surgeries during June 2015 to December 2018, in 3 specialized centers of the Colombo district of Sri Lanka. None of the patients had suspicious clinical, radiological or cytological findings of thyroid malignancy. All the cases with a FNAC diagnosis of malignancy or suspicious of malignancy prior to surgery were excluded from the current study. The study sample included a total of 92 cases over a period of $31 / 2$ years. Clinical, radiological and FNAC diagnoses have been supportive of benign entities. Thyroidectomy specimens of the study sample have been dispatched to the laboratory immersed in an adequate volume of the formalin fixative. Following adequate fixation gross details have been documented and the specimens were serially sliced, 3 -4 mm apart. Representative tissue sections were submitted for routine tissue processing and staining with hematoxylin and eosin. Histological diagnoses were reviewed independently by 2 consultant pathologists. Identification details of the patients were concealed by using a coding system.

\section{RESULTS AND DISCUSSION}

The study group included 92 cases of thyroidectomies. There were 81 cases of females and 11 cases of males. Mean age for females was 56 and males 62 years (table 01).

Table 1: Age \& sex distribution of thyroidectomy specimens -

\begin{tabular}{lll}
\hline $\mathbf{n = 9 2}$ & Gender & Mean age (years) \\
\hline Females & 81 & 56 \\
Males & 11 & 62 \\
\hline
\end{tabular}

Table 2: Thyroid profile (euthyroid, hypothyroid, hyperthyroid)

Thyroid profile $(\mathbf{n}=92)$

Euthyroid - 67 Hypothyroid - 14 Hyperthyroid - 11

The thyroid profile of the cases revealed that the majority of patients $(72.82 \%)$ in the study group were euthyroid (table 02).

Histological findings of the thyroidectomy specimens submitted for presumed benign diseases are shown below (figure 01). There were $9.78 \%$ cases of incidental thyroid carcinoma. 


\section{Thyroidectomy findings}
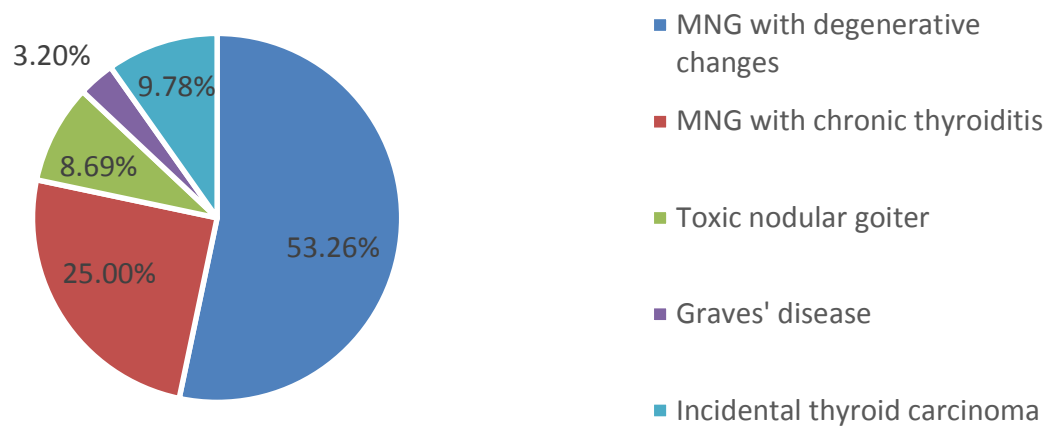

Figure 1: Thyroidectomy results

MNG with degenerative changes- $49(53.26 \%)$

MNG with chronic thyroiditis - $23(25 \%)$

Toxic nodular goiter - 08 (8.69)

Graves' disease - 03 (3.2\%)

Incidental thyroid carcinoma - 09 (9.78\%)

Table 3: Incidental thyroid carcinoma - histological type, stage and associated pathology

\begin{tabular}{|c|c|c|c|c|c|}
\hline \multicolumn{6}{|c|}{ Incidental thyroid carcinoma $(n=9)$} \\
\hline Туре & & Size & Multimodality & Stage & $\begin{array}{l}\text { Associated } \\
\text { pathology }\end{array}$ \\
\hline \multirow{3}{*}{$\begin{array}{l}\text { Papillary } \\
\text { microcarcinoma } \\
\text { (conventional type) } \\
\text { Follicular variant of } \\
\text { papillary carcinoma }\end{array}$} & 04 & $<10 \mathrm{~mm}$ & Absent & pT1 & Chronic thyroiditis \\
\hline & 04 & $<10 \mathrm{~mm}$ & Present $(n=1)$ & pT1 & Chronic thyroiditis \\
\hline & 01 & $22 \mathrm{~mm}$ & Absent & pT2 & Toxic goiter \\
\hline
\end{tabular}

There were 04 cases of papillary microcarcinoma exhibiting the conventional pattern and 05 cases of follicular variant of papillary carcinoma (table 03).

\section{DISCUSSION:}

The study reviewed 92 cases of thyroidectomy samples in 3 different specialized surgical units. In this cohort the mean age for females was 56 years and males was 62 years (table 01). The majority of patients were euthyroid $(n=67 ; 72.82 \%)$. There were 14 cases with hypothyroidism and 11 cases with hyperthyroidism (table 02). Review of histological diagnoses revealed that the majority of cases had benign pathological entities $(n=83)$. However, there were 09 cases of incidental thyroid carcinoma (figure 1). This was an unexpected finding in the cohort of 92 cases. Nine cases of incidental thyroid carcinoma did not have suspicious clinical, radiological or cytological findings prior to surgery. 
Moreover, the macroscopic assessment of the thyroidectomies with incidental carcinoma has shown solid, whitish lesions that were $<10 \mathrm{~mm}$ in diameter in eight out of 9 cases. In one case the tumour was $22 \mathrm{~mm}$ in diameter (pT2). Subsequent histological evaluation has confirmed the presence of incidental thyroid carcinoma. In one out of nine cases the tumour was multifocal and had 2 separate nodules involving both lobes. All nine cases were papillary carcinomas ( $\mathrm{p}$ $<0.05)$ and 4 tumours showed the conventional pattern while the remaining were classified as follicular variant of papillary carcinoma (table 03).

In eight cases of incidental thyroid carcinoma, the non-neoplastic thyroid tissue revealed features of chronic thyroiditis ( $\mathrm{p}$ <0.05). One case was associated with toxic features. Other pathological features such as degenerative changes and calcification did not show a statistically significant association with incidental thyroid carcinoma. None of the tumours had vascular or capsular invasion. There was no evidence of extracapsular spread in any of the cases of incidental carcinoma. A retrospective study conducted by Gandolfi P P, Frisina A, et al, have shown interesting results about incidental carcinoma in multinodular goiters (13). The multiinstitutional study done by Smith JJ et al (14), has also shown very similar results with regard to incidental thyroid carcinoma. Authors have described the risk factors of incidental thyroid carcinoma. Paolo Miccoli et al have also conducted a comprehensive study on the above topic and discussed in detail about the increased frequency of incidental thyroid carcinoma (15).

\section{CONCLUSION:}

Thyroid diseases are becoming more prevalent in many parts of the world. In Sri Lanka thyroid cancer is the second most common malignancy in females. In a cohort of 92 patients who underwent thyroidectomy surgery for presumed benign diseases such as MNG, chronic thyroiditis, toxic nodular goiter and Graves' disease, the finding of thyroid cancer was an unexpected event. In the present study there were 09 cases $(9.78 \%)$ of incidental thyroid carcinoma that was histologically confirmed as papillary type $(\mathrm{p}<0.05)$, which is the most common group of thyroid malignancy. Eight out of 9 cases were $<10 \mathrm{~mm}$ in maximum diameter (microcarcinoma), and have not been picked up by the FNAC technique owing to the small size. Radiological studies have not been able to identify the focal lesion due to the varied vascular pattern and degenerative changes seen in MNG. There was a single case of incidental carcinoma which was $>20 \mathrm{~mm}$ in diameter (pT2), and associated with a toxic goiter. Chronic thyroiditis showed a statistically significant association with incidental thyroid carcinoma $(\mathrm{p}<$ $0.05)$.

With limited facilities for ultrasound scanning of each and every MNG especially in a resource poor setting, there is a risk of missing microcarcinomas by FNAC technique. External examination in isolation is not a reliable way of determining the suspicious nodules for the 
FNAC procedure. We hope a close collaboration between pathologist, radiologist, surgeon and endocrinologist would help to improve the diagnostic accuracy and steer towards the most appropriate management option for the patient. All the patients with incidental carcinoma in the present study have been followed up with imaging studies and serum thyroglobulin assay with no evidence of local or systemic disease up to date.

\section{REFERENCES:}

1. Frilling A, Liu C, Weber F. "Benign multinodular goiter". Scandinavian Journal of Surgery. 2004; 93 (4): 278-81. doi:10.1177/145749690409300405. PMID 15658668.

2. Khatawkar A V, Awati S M. Multi-nodular goiter: Epidemiology, Etiology, Pathogenesis and Pathology. International Archives of Integrated Medicine. 2015; 2(9): 152-156. http://imsear.searo.who.int/jspui/handle/123456789/164959

3. Pinchera A, Aghini-Lombardi F, Antonangeli L, Vitti P. Multinodular goitre. Epidemiology and prevention. Ann Ital Chir. 1996;67:317-25.

4. Cancer Incidence Data, National Cancer Control Program, Sri Lanka 2014. nccp.health.gov.lk

5. Vanderpas J. Nutritional epidemiology and thyroid hormone metabolism. Ann Rev Nutr. 2006;26:293-322.

6. Knudsen N, Laurberg P, Perrild H, Bülow I, Ovesen L, Jørgensen T. Risk factors for goiter and thyroid nodules. Thyroid. 2002;12:879-88.

7. WHO/UNICEF/ICCIDD. Assessment of iodine deficiency disorders and monitoring their elimination. A guide for program managers. 3rd edn. Geneva: WHO, 2007.

8. Zimmermann MB. Iodine deficiency. Endocr Rev 2009;30:376-408.

9. Hagag P, Strauss S, Weiss M. Role of ultrasound guided fine needle aspiration biopsy in evaluation of non-palpable thyroid nodules. Thyroid 1999;8:989-95.

10. Braga M, Cavalcanti TC, Collaco LM, Graf H. Efficacy of ultrasound-guided fineneedle aspiration biopsy in the diagnosis of complex thyroid nodules. J Clin Endocrinol Metab. 2001;86:4089-91.

11. Tan G, Gharib H. Thyroid incidentalomas: management approaches to nonpalpable nodules discovered incidentally on thyroid imaging. Ann Intern Med. 1997;126:22631.

12. Cibas E, Syed A. The Bethesda system for reporting thyroid cytopathology. Am J Clin Pathol 2009; 132:658-65.

13. Gandolfi P P, Frisina A, Raffa M, Renda F, Rocchetti O, Ruggeri C, Tombolini A. "The incidence of thyroid carcinoma in multinodular goiter: Retrospective analysis". Acta Bio-medica : Atenei Parmensis.2004;75 (2): 114-7. PMID 15481700. 
14. Smith JJ et al. Cancer after thyroidectomy: a multi-institutional experience with 1,523 patients. J Am Coll Surg. 2013;216:571-9.

15. Paolo Miccoli, Michele N Minuto, David Galleri, Jacopo D’Agostino, Fulvio Basolo, Lucia Antonangeli. Incidental thyroid carcinoma in a large series of consecutive patients operated on for benign thyroid disease. ANZ Journal of Surgery. 2006;76(3): 123-126. https://doi.org/10.1111/j.1445-2197.2007.2006.03667.x

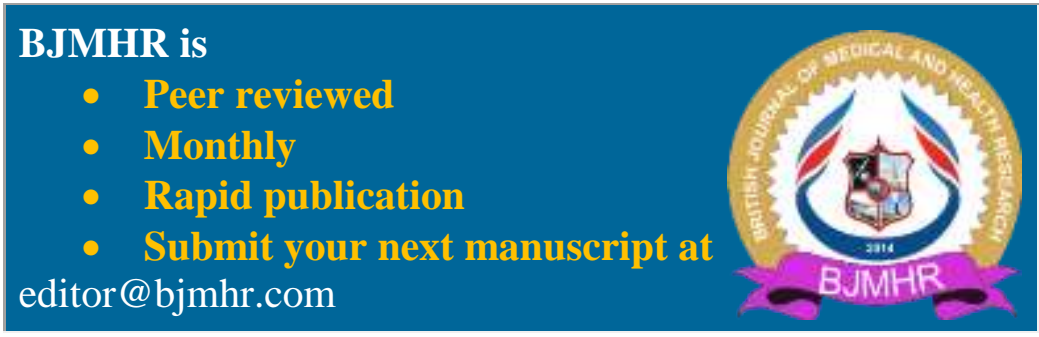

